\title{
La libéralisation des chemins de fer européens au regard de l'histoire
}

Table ronde animée par Patrice Leroy. Introduction historique par Michèle Merger

The liberalization of European railways from a historical pe rspective

Patrice Leroy, Michèle Merger, Yves Crozet, Michel Lebœuf, Hubert du Mesnil et Émile Quinet

\section{OpenEdition}

\section{Journals}

Édition électronique

URL : https://journals.openedition.org/rhcf/889

DOI : $10.4000 /$ rhcf.889

Éditeur

Rails \& histoire

Édition imprimée

Date de publication : 1 juin 2008

Pagination : 147-176

ISSN : 0996-9403

Référence électronique

Patrice Leroy, Michèle Merger, Yves Crozet, Michel Lebœuf, Hubert du Mesnil et Émile Quinet, «La

libéralisation des chemins de fer européens au regard de l'histoire ", Revue d'histoire des chemins de fer [En ligne], 39 | 2008, mis en ligne le 01 juin 2011, consulté le 22 avril 2022. URL : http:// journals.openedition.org/rhcf/889; DOI : https://doi.org/10.4000/rhcf.889 


\section{TABLE RONDE}

\section{La libéralisation des chemins de fer européens au regard de l'histoire}

Animateur : M. Patrice Leroy, directeur honoraire de la SNCF, ancien secrétaire général adjoint du CEEP, président délégué de I'AHICF

Introduction historique : Madame Michèle Merger, chargée de recherche au CNRS, membre du Comité scientifique de I'AHICF

Avec :

M. Yves Crozet, professeur à I'université Lumière-Lyon 2 M. Michel Lebœuf, directeur Développement à Voyageurs France Europe, SNCF M. Hubert du Mesnil, président de RFF

M. Émile Quinet, professeur émérite à l'École nationale des Ponts et Chaussées

\section{Patrice Leroy}

Dans l'esprit d'associer l'histoire à des sujets d'actualité brûlants, cette table ronde aborde la libéralisation des chemins de fer européens au regard de l'histoire. Après que chacun des participants se sera présenté, je donnerai la parole à Michèle Merger pour une introduction historique, puis chacun interviendra avant d'élargir le débat.

\section{Michèle Merger}

Chargée de recherche au CNRS, à l'Institut d'histoire moderne et contemporaine, et membre du Comité scientifique de l'AHICF, j'ai eu le plaisir de diriger un projet de recherche européen d'histoire au temps présent, l'action COST 340 «Vers un réseau de transport européen intermodal : les leçons de l'histoire ». Mes recherches récentes portent sur les grands projets transeuropéens tels le Lyon-Turin, et la libéralisation des chemins de fer européens depuis les années 1990. 


\section{Émile Quinet}

Je suis professeur émérite à l'École nationale des Ponts et Chaussées, spécialisé en économie des transports et, également, membre du Comité scientifique de l'Association.

\section{Michel Lebœuf}

Je suis le directeur du développement du secteur Voyageurs FranceEurope, la branche de la SNCF chargée du transport des voyageurs à longue distance, aussi bien pour le transport intérieur que pour le transport international. Dans ma fonction, je suis chargé d'instruire tous les projets de développement à long terme, ainsi qu'un certain nombre des sujets stratégiques qui ont un lien assez étroit avec cette table ronde, comme la politique de péage avec l'État et RFF et la stratégie européenne.

\section{Hubert du Mesnil}

Je suis président de Réseau ferré de France après en avoir été le directeur général. Ni historien, ni spécialiste du chemin de fer, j'ai été cependant à la fois témoin et acteur de l'histoire de ces dix dernières années, puisque, avant un passage dans le monde du transport aérien, j'ai été directeur au ministère des Transports, notamment dans la période 1995-2000, qui a connu des étapes qui ont marqué l'histoire récente.

\section{Yves Crozet}

Yves Crozet, professeur à l'université de Lyon et membre du Laboratoire d'économie des transports.

\section{Michèle Merger}

Monsieur le Président, Mesdames et Messieurs, je voudrais rappeler dans cette courte introduction que, en mai 1997, l'Association pour l'histoire des chemins de fer en France avait déjà organisé un colloque, le cinquième pour elle, autour d'un thème, brûlant à l'époque, «Le chemin de fer en temps de concurrence : choix du XIX ${ }^{e}$ siècle et débats actuels ». Ce projet avait été conduit sous la direction scientifique de Michel Walrave, ici présent, et sous la mienne. Il visait à mettre en regard les longs débats qui avaient accompagné la construction des premières lignes de chemin de fer et ceux qui étaient apparus au début des années 1990 et, plus précisément, au lendemain de la publication de la célèbre directive de la Commission européenne, la directive 1991-440, de juillet 1991, qui concernait le développement des chemins de fer communautaires. 
Dix ans se sont écoulés depuis cette rencontre durant laquelle une table ronde avait été organisée et animée par François Caron sur le thème «Infrastructures et exploitation ferroviaire : intégration ou séparation? Les enjeux d'une réforme au regard de l'histoire»'. Le débat avait donc porté sur l'un des quatre changements fondamentaux préconisés par la directive 91/440, les trois autres étant l'indépendance de gestion des entreprises ferroviaires, leur assainissement financier et le libre accès à l'infrastructure.

Dans son introduction, M. Caron avait rappelé, fort justement, comment les logiques de l'intégration de l'infrastructure et de l'exploitation s'étaient imposées historiquement. Je dis bien «intégration de l'infrastructure et de l'exploitation ». «Le chemin de fer, écrivait-il, était vite perçu comme étant un système global, où l'interdépendance des trois grands services, Voie et Bâtiments, Matériel et Traction et Exploitation était si étroite qu'elle les rendait inséparables. »Cette intégration a été justifiée par la théorie du monopole naturel dont le corollaire était le concept juridique de service public. Ces concepts et cette réalité ont été renforcés avec la nationalisation progressive des réseaux au cours de la première moitié du $\mathrm{XX}^{\mathrm{e}}$ siècle, à la suite de la nationalisation des chemins de fer suisses intervenue à la fin du XIX ${ }^{e}$. Cette directive était la première d'une longue série visant à recomposer le transport ferroviaire de l'Union en fonction d'une approche libérale et en s'inspirant de l'exemple de la Suède qui avait commencé de réformer son système ferroviaire dès le début de $1989^{2}$. Il s'agissait de mettre fin au statut juridique des entreprises publiques ferroviaires et au monopole de l'exploitation dont elles bénéficiaient et dont elles avaient hérité lors de la nationalisation des réseaux de chaque pays. Il s'agissait également d'ouvrir ces derniers à la libre concurrence afin de faciliter leur adaptation aux conditions du marché unique, d'accroître leurs activités et d'améliorer la qualité de leurs services.

Ces principes étaient déjà contenus, affirmés et signés par les premiers membres de l'Union, ou plutôt de la Communauté européenne. Le Traité de Rome, c'était bien l'ouverture des marchés et l'ouverture des réseaux, la libre circulation.

La table ronde d'aujourd'hui ne prétend pas dresser un bilan complet de cette « thérapie de choc » européenne qui s'appuie sur l’idée «que l’utilité sociale fondée sur la concurrence est plus grande que l'utilité sociale issue du monopole

1- Voir «Les chemins de fer en temps de concurrence. Choix du XIX siècle et débats actuels », Revue d'bistoire des chemins de fer, $\mathrm{n}^{\circ} 16-17$ (printemps-automne 1997), p. 392-405 et la reproduction de ce texte, ci-après, p. 177.

2- Voir la chronologie en annexe.

3- Expression utilisée par le ministre des Transports luxembourgeois Henri Grethen pour désigner la libéralisation du trafic ferroviaire de marchandises, Rail et transports, 9 avril 2003, p. 16. 
naturel ${ }^{4}$, mais cette « thérapie » - car malgré les retards accumulés, dus aux difficultés d'application de ces directives et aux vives oppositions que suscitait cette politique de libéralisation, nous pouvons affirmer qu'au cours des quinze dernières années les chemins de fer européens ont davantage évolué que durant les quatre décennies précédentes - me conduit à dégager plusieurs questions auxquelles sont invités à répondre les différents intervenants de cette table ronde.

Une première question se pose et vient à l'esprit tout naturellement : «Quels sont les pays et les acteurs européens les plus réfractaires à cette thérapie ? » et, à l'inverse, "Quels sont les pays et les acteurs les plus favorables ?» Pour les premiers, c'est-à-dire les réfractaires, quels arguments opposent-ils aux directives de Bruxelles?

La deuxième question : «Existe-t-il une typologie des réformes entreprises, comme il y avait eu des modèles du point de vue de l'organisation, et donc, du point de vue de la concession du statut juridique des différentes compagnies privées au XIX ${ }^{\mathrm{e}}$ siècle ? N'y a-t-il pas là aussi des modèles, et si oui, quels sont-ils?»

Troisième question : «Quel type de service ferroviaire attendons-nous et pouvons-nous attendre... ? » En d'autres termes, quel est le devenir du concept de service public ? Va-t-il être remplacé par le concept de service universel qui a été défini par une directive européenne de 1996 et qui concerne les télécommunications et la poste, deux entreprises de réseaux, soumises elles aussi à la politique de libéralisation préconisée par Bruxelles ?

Enfin, la quatrième et dernière question : «Qu'en est-il de la revitalisation du fret ferroviaire au lendemain de l'ouverture des corridors de fret, accessibles à des opérateurs tiers, et favorisés par ces directives, notamment des ensembles des paquets ferroviaires : le premier et le second notamment ?» La part des chemins de fer dans le cadre du trafic des marchandises est-elle tendanciellement orientée à la baisse ? A-t-elle oui ou non résisté à l'érosion là où les réformes sont plus approfondies? Aussi, quels sont les nouveaux entrants et quelle est la nouvelle Europe du fret ferroviaire?

\section{Patrice Leroy}

Merci, Michèle Merger. Je vais d'abord donner la parole à Hubert du Mesnil. Comment se positionne RFF et quelles sont vos priorités sur ce sujet?

4- F. Caron, «Table ronde Infrastructures et exploitation ferroviaire... », art. cit., p. 394, ciaprès p. 177. 


\section{Hubert du Mesnil}

Le sujet est vaste, il faut l'aborder avec modestie, d'autant qu'il y a dans cette salle des personnalités qui ont pris une part déterminante dans cette histoire récente et qui mériteraient autant que moi de s'exprimer à son sujet. J'ai envie de m'écarter de la voix officielle d'une entreprise qui a sur ces sujets des positions, des doctrines... pour apporter un témoignage personnel de ce que je ressens, que j'ai observé, et que je vois à travers la vision d'un gestionnaire d'infrastructures qui tente de trouver sa place dans le système.

Vous posez la question de manière un peu rude : « les pays réfractaires »... Vous savez qu'à Bruxelles tout le monde considère que la France fait partie des pays les plus réfractaires, et son image, je dis bien «image», est celle d'un pays complètement réfractaire.

Quant à «la thérapie de choc », c'est l'expression employée par un ministre luxembourgeois, or on ne peut pas dire que ce ministre luxembourgeois ait appliqué cette thérapie de choc à sa propre entreprise ferroviaire. Parfois, dans les discours, on se laisse un peu aller, on utilise des mots qui plaisent ou qui frappent. Ce qui est sûr, c'est que les chemins de fer en Europe sont tous entrés en crise les uns après les autres, ou les uns et les autres; et ils ont tous été menacés dans une concurrence intermodale où ils ont régressé très fortement par rapport aux modes montants. Tous ont dû trouver d'une manière ou d'une autre une thérapie dans laquelle ont été combinés des réformes, des changements qui ont associé des réformes de structure, des modalités de financement, des changements de personnes, une dose de libéralisation, une dose d'Europe. Les corps, si je puis dire, étaient malades, la thérapie nécessaire. Elle a été diversifiée. Je ne suis pas certain qu'elle se résume purement et simplement à l'introduction de la libéralisation, c'est-à-dire à l'ouverture du marché. Je pense que plusieurs registres ont été utilisés différemment par chacun des pays. En termes d'image, il est clair que nous faisons partie des pays qui n'ont pas cru à la libéralisation, ou qui n'ont pas voulu pendant certaines périodes aborder le problème sous cet angle. Je pense que la France comptait parmi les opposants à l'adoption de ces directives, qu'elle y a longtemps résisté sur le plan politique : des gouvernements successifs de droite et de gauche ont tenté de s'y opposer. J'ai participé à une étape de cette négociation européenne, celle du paquet ferroviaire négocié entre 1997 et 2000, à l'époque où Jean-Claude Gayssot était ministre des Transports et où la France, notamment lorsqu'elle assurait la présidence de l'Europe, a eu l'occasion de d'orienter cette négociation européenne. L'une des raisons pour lesquelles on s'y est opposé et l'une des raisons pour lesquelles nous étions considérés comme réfractaires, c'est que nous ne voulions pas que la thérapie de choc se résume à une simple ouverture à la concurrence. Nous avons voulu faire valoir l'idée que le redressement du 
mode ferroviaire en Europe consistait aussi à élargir le réseau à l'échelle de l'Europe. Construire un réseau européen, c'est traiter de questions d'interopérabilité, d'harmonisation technique, d'harmonisation tarifaire, d'harmonisation des règles de concurrence par rapport aux autres modes, mais ce n'est en aucun cas une simple question d'ouverture à la concurrence. En ce sens, nous faisions effectivement partie des réfractaires. D'autres formes de réticences se sont exprimées : je n'ai par exemple jamais eu l'impression, à l'époque, que nos collègues luxembourgeois étaient des militants de l'ouverture, bien qu'ils aient été plutôt libéraux. À l'échelle du Luxembourg, la notion de l'ouverture à la concurrence n'était peut-être pas la solution à leurs difficultés ; le problème était sans doute le même pour la Grèce ou l'Irlande. Ces concepts n'étaient pas forcément les mieux adaptés à leur situation. Nous voulions simplement agrandir le champ de vision, en disant qu'il paraissait utile d'élargir le sujet aux autres aspects de l'évolution ou du redressement du secteur ferroviaire et de la construction d'une Europe ferroviaire qui ne pouvait pas se contenter d'être ramenée à une Europe concurrente.

Il est vrai que le cheminement a été très différent suivant les situations : chaque pays a entrepris le sien, jusqu'à des exemples un peu caricaturaux... L'Angleterre, à sa manière, a opté pour la thérapie de choc et l'on peut en parler comme d'une voie ultralibérale perceptible dès le début, puis qui a trébuché et qui, finalement, a connu des évolutions très substantielles. On s'est singulièrement écarté du modèle initial sur un certain nombre de points. On peut dire d'une certaine manière que la France a dit «non » à la concurrence pendant un certain temps, puis s'y met à sa manière. Si aujourd'hui on regarde ce qui est fait concrètement en matière d'accueil des nouveaux entrants, je ne suis pas certain que la France réserve le moins bon accueil aux nouvelles entreprises ferroviaires; je ne suis pas certain que nous soyons le plus mauvais élève de la classe quand il faut délivrer un certificat de sécurité ou permettre à une entreprise anglaise ou belge de venir chez nous. Je constate que, en dix-huit mois, toutes les régions de France sans exception sont aujourd'hui traversées par des entreprises concurrentes, ou de "nouveaux entrants », comme on les appelle. Toutes, sans exception. Et je ne suis pas certain que ce taux de pénétration des entreprises étrangères sur le marché français soit honteux par rapport à celui qu'ont connu les autres pays. Mais alors, pourquoi la France est-elle considérée comme réfractaire ? Parce que notre démarche a été particulière, qu'elle a été progressive, qu'il y a un certain nombre d'instruments du libéralisme que nous n'avons pas voulu mettre en place tout de suite : je pense à la création du régulateur. Il est clair que, au regard du concept, le fait qu'il n'existe pas de régulateur en France est considéré comme une abomination. Il n'existe pas de régulateur, certes, mais il y a quand même un pays, une 
administration qui assure la régulation, et cela n'empêche pas l'arrivée des nouveaux entrants. En pratique, chaque réforme a été menée à sa manière et, plus le temps passe, plus on s'aperçoit, me semble-t-il, que ces réformes ont été menées en tenant compte d'un contexte local, d'une histoire locale, d'un dimensionnement, d'une géographie, d'une sociologie : le Luxembourg, la France ou l'Espagne, ce n'est pas la même chose. Mais on peut aussi constater que, au fil du temps, plus les étapes se succèdent, conduites par chacun à sa manière, plus il existe d'éléments de convergence qui nous rapprochent progressivement les uns des autres, par des cheminements complètement différents. Je ne serais pas loin de penser que, dans quelques années, la situation ferroviaire s'harmonisera, qu'il existera un marché européen, que sur ce marché européen il y aura un certain nombre de grandes entreprises historiques qui auront constitué des places à l'échelle de l'Europe et des positions majoritaires, des systèmes dominants, auxquels il faudra ajouter l'apparition d'un certain nombre d'acteurs nouveaux, très diversifiés : des petits, des moyens et des grands, certains proches des armateurs, d'autres proches des grands chargeurs, peu importe... Mais il me semble que, par des cheminements très différents, et très diversifiés, on peut imaginer que, dans quelques années, finalement, il y aura un rapprochement et que les choses s'harmoniseront peu à peu.

En ce qui concerne le service public ferroviaire, je préférerais laisser la SNCF et M. Lebœuf en parler. Pour nous, il ne fait aucun doute que l'infrastructure est un service public. Nous sommes dans le monopole ou le quasi-monopole, par conséquent, dans le service public, l'intérêt public... On verra s'il faut revenir sur l'idée de la privatisation de l'infrastructure. Personnellement, je ne le crois pas. Vous avez vu ce qui s'est passé en Angleterre ; des débats intéressants ont lieu en ce moment avec l'Allemagne où la question se pose de savoir si, dans la privatisation de la DB, on inclut ou non l'infrastructure. Curieusement, des personnalités politiques d'Allemagne viennent nous demander comment ça se passe en France, parce qu'ils se demandent si, finalement, ce que nous avons fait en France, qui paraissait très obscur, très antilibéral..., était si bête que ça.

Terminons avec la question du fret. Il est vrai que, pour le fret, on ne peut pas ne pas se poser la question du rapprochement entre l'ouverture et la revitalisation. Cette évolution nous interpelle : nous devons comprendre pourquoi le monopole français a pu engendrer le TGV et pourquoi il n'a pas pu engendrer quelque chose d'équivalent pour le fret. La question qui s'ensuit est purement théorique : si nous étions restés dans le monopole, auquel le retour n'est plus possible, aurions-nous pu redresser le fret ferroviaire ? Pourquoi un monopole est-il capable, dans un cas d'engendrer une telle puissance d'innovation et un tel succès commercial, et dans un autre de s'avérer, apparemment, 
impuissant à le faire ? Puisque, au moins jusqu'à l'année dernière, toutes les démarches successives, tous les efforts successifs, considérables du côté de la $\mathrm{SNCF}$, n'ont pas permis d'opérer ce redressement, alors qu'apparemment, là où les marchés se sont ouverts pour le fret ferroviaire, des résultats ont été obtenus qui semblent réellement meilleurs que chez nous?

\section{Patrice Leroy}

Pour faire alterner la parole des acteurs et celle des chercheurs, je donne la parole à Yves Crozet. Les questions qui sont posées ne donnent-elles pas des repères, historiques ou d'une autre nature?

\section{Yves Crozet}

Le premier repère, le premier qu'il faut traiter, c'est la schizophrénie française. L'expression « directive » prête en effet à confusion, parce qu'on a vraiment l'impression que c'est un oukase qui tombe du ciel de Bruxelles, alors que, comme l'a expliqué Hubert du Mesnil, ces directives ont été négociées par la France, et que la France les a acceptées. Quand une directive vient de Bruxelles, elle a été discutée avec les élus, par exemple avec M. Gayssot. Pour bien le comprendre, il suffit de regarder ce qui a été fait dans les différents pays et qui est extrêmement différent. Une fois que l'on accepte le principe de la séparation de l'infrastructure et de l'exploitation, que peut-on faire ? la séparation comprend au moins quatre degrés sur l'axe de l'infrastructure. La séparation comptable des deux secteurs d'abord, comme le font les Allemands ou les Suisses. En allant plus loin, on conserve comme la France un monopole public; au-delà, une première étape est la création d'un monopole privé et la dernière un retour au XIX ${ }^{e}$ siècle, avec une multitude de propriétaires de l'infrastructure différents.

Sur l'axe de l'exploitation, soit vous gardez, comme la France, un seul opérateur ferroviaire, au moins jusqu'à l'ouverture du fret ; soit vous gardez un opérateur dominant, comme en Allemagne, avec quelques concurrents dont Keolis qui vient d'emporter un nouveau marché ; soit vous allez encore plus loin, comme les Anglais : vous faites un allotissement du réseau à 25 opérateurs ; soit, carrément, vous rêvez à l'open access avec des milliers d'opérateurs, de la concurrence dont nous, économistes, nous rêvons.

Vous le voyez, avec quatre options sur le premier axe et quatre sur le second vous obtenez seize possibilités. Évidemment, le sens de l'histoire n'est pas d'aller à la case extrême qui aboutirait à la fois à une multitude d'opérateurs et à une multitude de propriétaires. Peut-être que quelques économistes en ont rêvé, mais ce n'est pas du tout le résultat espéré. Ce résultat n'est pas non plus le statu quo, ou le retour d'un monolithe ferroviaire intégré. Le 
résultat, si vous regardez les modèles allemand, français, suédois, et même anglais, c'est qu'on revient partout au même système : une infrastructure globalement gérée par les puissances publiques, d'où les questions posées par les Allemands, parce qu'ils seraient les seuls en Europe à prendre le risque de la privatisation. Je vous rappelle que, en Allemagne, la demande commune d'une privatisation du réseau vient du syndicat, parce qu'il veut éviter l'éclatement du personnel entre ceux qui gèrent le réseau et ceux qui gèrent l'exploitation. On reste partout ailleurs sur des logiques de propriété publique. Du côté de l'exploitation, le résultat est uniforme, comme il l'est dans les télécommunications, dans le transport aérien, dans toutes les industries de réseau. On voit apparaître quelques gros opérateurs dominants et des opérateurs secondaires. On voit bien que, de fait, après une vingtaine d'années de réformes, le système converge vers une configuration qui existe dans les autres industries de réseau.

Mais le paradoxe français, c'est que notre schizophrénie nous pousse à ne pas voir la situation, à renâcler, à rechigner, comme si ces réformes nous avaient été imposées, alors que nous les avons acceptées et négociées. Or dans ce jeu-là, nous sommes gagnants! C'est un grand paradoxe des Français : comment vous, nous, Français, sommes vus par les Anglais, par les Allemands, etc. ? Nous sommes vus en permanence comme des traitres, des râleurs, voire pire... Mais de ceux qui se sortent de la situation, qui, finalement, s'en sortent très bien. Je suis extrêmement optimiste sur la situation du groupe SNCF à l'horizon de dix ou quinze ans dans le paysage européen. Reste la partie «fret » qui pose problème, mais je suis extrêmement optimiste sur la position des opérateurs français et de Veolia Transport qui est aussi un opérateur français de fret. Je suis très optimiste parce que, ce qui a changé fondamentalement dans les réformes, c'est que nous ne nous posons plus de questions à l'échelle de la nation, mais des questions d'entreprise. On raisonne en termes d'optimisation d'entreprise et c'est évidemment ce qui pose question à nos traditions, à nous, Français : l'indicateur de profit devient un indicateur de performance collective, et pas seulement de performance privée. Le profit, c'est le signe qu'on apporte du surplus à la collectivité, alors que les pertes et les subventions, c'est le signe qu'on prend du surplus à la collectivité, c'est là l'immense changement. Par conséquent, aujourd'hui, ce que la collectivité demande à la SNCF et aux autres opérateurs privés, c'est qu'ils gagnent de l'argent, parce que, s'ils en perdent, cela signifie que la collectivité doit payer pour eux. C'est un changement important, au-delà même du mot « libéralisation », parce que la SNCF peut rester propriété publique, un EPIC, pendant de nombreuses années encore, il n'y a aucune raison de la privatiser. Ce qui change la donne, c'est que cette société, on lui a donné comme objectif de ne plus faire de déficits, puisqu'on l'a déchargée d'une partie de la dette et que les péages ont 
été mis à des niveaux tels - de cela, vous débattrez entre vous -, qu'on n'a pas tué la SNCF. La preuve : son bénéfice récent. Le système est là pour que ce soit bien l'indicateur du profit qui soit l'indicateur collectif de bien-être, et c'est en cela que les Français ont un gros problème.

\section{Patrice Leroy}

Merci, Yves Crozet. « Passer d'un raisonnement de nation à un raisonnement d'entreprise » est une belle formule. Michel Lebœuf, comment ressens-tu les choses de l'intérieur de la SNCF ?

\section{Michel Lebœuf}

Je vais répondre aux questions qui ont été posées, d'abord en ce qui concerne la «thérapie de choc ». Je pense que, de ce point de vue, on peut dire qu'en France on a fait la réforme au minimum. C'est peut-être pour cela que certains nous considèrent comme des réfractaires. Effectivement, nous avons séparé juridiquement l'infrastructure de l'exploitation ferroviaire. Ce n'est pas en apparence une réforme minimum, sauf que le gestionnaire de l'infrastructure n'a pas les forces nécessaires pour entretenir ou renouveler les voies. Il passe un contrat avec la SNCF qu'on n'a pas voulu démanteler ou séparer en plusieurs entités pour des raisons qu'on imagine. On a essayé de faire bouger les choses, mais au minimum. Il en va de même dans un autre domaine, parce qu'il faut rappeler que la directive 1991-440 n'est pas qu'une directive de libéralisation. Elle suggère aussi que les États essayent de désendetter le secteur ferroviaire. Je pense qu'en France on était plutôt dans une solution minimum, par comparaison, par exemple, avec l'Allemagne.

De ce point de vue, on peut dire que « oui, effectivement, la France a été plutôt réfractaire ». Mais maintenant, quand on regarde ce qu'ont fait les autres pays, notamment l'Allemagne qui dit: «Moi, j’ai ouvert mon marché très vite, etc. », et quand on regarde justement le fret, ceci m'amène à répondre à l'une de vos questions... Le fret a été libéralisé en Allemagne dès 1994, alors que chez nous il le fut seulement en 2006. Mais il a fallu attendre six ans en Allemagne pour que les nouveaux entrants atteignent $3 \%$ du marché ferroviaire. Aujourd'hui (en 2006), ils ont à peu près entre 16 et $17 \%$ du marché. Nous, environ en deux ans, nous sommes aujourd'hui à $3 \%$. On peut considérer qu'effectivement, je souscris à ce qu'a dit le président Hubert du Mesnil, je crois que de ce point de vue, on a mis en place les conditions qui permettent d'accueillir correctement les nouveaux entrants. Peut-être trop correctement de l'avis de la SNCF, mais en tout cas, il est clair que les nouveaux entrants sont en passe de prendre des parts de marché significatives. Nous avons calculé, 
par exemple, que la capacité de transport actuelle des nouveaux entrants représente à peu près $10 \%$ du trafic fret. Aujourd'hui, ils sont trois, ils ne sont pas en pleine utilisation de leurs capacités. Il y aura de d'autres nouveaux entrants ; actuellement, sept entreprises ont un certificat, des entreprises françaises, belges ou luxembourgeoises, Eurotunnel a même récemment créé un nouvel entrant. Il est vrai que la France est plutôt réfractaire. Quand je regarde par exemple le projet de loi pour la création d'une autorité de régulation, je trouve personnellement que c'est un projet minimum. On ne donne à cette autorité qu'une seule mission, celle de s'assurer de la non-discrimination. En réalité, ce régulateur, que l'on crée au dernier moment, est fondé sur l'article 30 de la directive 2001-14, lequel article dit que l'autorité doit aussi regarder si la tarification est bonne pour le marché, mais cette fonction ne figure pas dans la mission que l'on propose aux régulateurs. Là, on est bien dans un projet minimum.

En ce qui concerne la typologie des réformes, deux grands cas se distinguent. Le premier, c'est ce qu'on appelle la concurrence sur le marché, et le second, c'est la concurrence pour le marché. En Angleterre, on est franchement dans la concurrence pour le marché. Dans les autres pays, on est plutôt pour l'accès libre au réseau, sauf, évidemment, pour un certain nombre de services publics qui feront l'objet d'appels d'offres dans le cadre du futur règlement OSP, qui seront des appels d'offres pour le marché. En France, en tout cas, nous sommes bien, dans les grandes lignes, dans la concurrence sur le marché, pour les voyageurs comme pour le fret.

En ce qui concerne le service public, je pense qu'en France il relève manifestement du règlement OSP qui entrera en vigueur à partir de 2010 et qui permet à une autorité organisatrice de transport de définir le transport qu'elle souhaite voir réaliser et de le confier après appel d'offres à un opérateur ou de le confier directement; la procédure d'appel d'offres n'est pas obligatoire. Ce règlement permet aussi une période d'adaptation de dix ans. En France, pour les services régionaux, on peut s'attendre qu'à partir de 2010 certaines régions lancent des appels d'offres, à mon avis de façon très progressive et peu risquée. En d'autres termes, je ne crois pas qu'une région lance un appel d'offres pour la totalité des TER sur son territoire, elle le fera plutôt pour une ligne ou deux, histoire de mettre la SNCF un peu en concurrence, histoire aussi, peutêtre, de booster son opérateur historique préféré.

Je tente aussi de répondre à une question que Hubert du Mesnil a très bien posée : « Pourquoi donc le fret n'arrive-t-il pas à se relever ? » Si on prend l'année 2007 où l'on a commencé à sentir un petit frémissement... que, je le crains, la grève a annihilé... Très franchement, je ne sais pas répondre à la question... Mais je pense qu'un certain nombre de facteurs peut expliquer cela. 
D'abord, il me semble que la SNCF a fait un choix plus implicite qu'explicite d'investissement massif dans le transport des voyageurs. Elle donne la priorité aux voyageurs et témoigne, à mon sens, d'une ambition beaucoup plus forte dans le domaine des voyageurs, peut-être aussi poussée par la technique et le plaisir de la vitesse, un facteur important. Évidemment, il y a aussi un certain nombre de facteurs sociaux, mais il y a aussi le fait que le fret a toujours été le parent pauvre dans l'exploitation opérationnelle. Il est évident que, lorsque la question se pose de savoir si l'on accorde la priorité aux trains de fret ou aux trains de voyageurs, la priorité va toujours aux voyageurs. Il semble qu'actuellement on essaye de remédier à cela, puisque dans les propositions qui ont été faites en matière de péage, RFF pense que le train de fret ne paye pas son coût marginal. Pour RFF, évidemment, il n'est pas intéressant de donner à un train fret son sillon. L'idée qui vient, c'est de faire en sorte que RFF ait une recette au moins égale à son coût marginal par train, donc, avec un Shadow péage, de telle façon qu'on puisse effectivement, par tous les moyens - ce moyen-là, mais d'autres aussi -, mis en œuvre à l'intérieur de la SNCF, redonner au fret la place qu'il devrait avoir. Dans ce domaine, il me semble que la SNCF est en train d'opérer un tournant. C'est peut-être ce qui explique qu'on est en train de changer, car jusqu'à présent, il y avait mutualisation des ressources. Les conducteurs n'étaient pas forcément des conducteurs de fret d'un côté et des conducteurs de voyageurs de l'autre, les locomotives servaient aux deux. Il se trouve qu'avec l'évolution, les grandes lignes ce sont les TGV. Par définition, leurs locomotives ne peuvent pas servir à faire du fret. Progressivement, on s'oriente vers une allocation des moyens. Et l'allocation des moyens, c'est quand même un des grands éléments de la réforme de l'outil industriel de la SNCF. Voilà un changement. Mais j’ajouterai que la concurrence en soi est elle aussi un élément très important, parce que, d'une part, elle opère une pression sur les prix qui est plutôt favorable pour le marché et, d'autre part, je pense que les cheminots avaient besoin d'être un peu réveillés; le fait de voir les nouveaux entrants, le fait de voir finalement leur activité en danger a, en ce sens, un certain nombre de vertus. On ne peut pas non plus ne pas évoquer la concurrence avec la route. Cette concurrence a-t-elle été favorable ou défavorable ? Je pense qu'un débat va s'instaurer, mais il n'empêche que dans le climat actuel, où l'énergie commence à coûter cher, et où la conscience des dégâts faits à l'environnement est de plus en plus forte, ces facteurs sont favorables à un essor du fret ferroviaire. Voilà ce que je voulais dire dans un premier temps pour apporter quelques éléments de réponse. 


\section{Émile Quinet}

C'est l'avantage et l'inconvénient d'intervenir en dernier : beaucoup de choses ont été abordées et ce que je vais dire va forcément être parcellaire pour apporter des remarques complémentaires à certains des points qui ont été évoqués.

En ce qui concerne le caractère réfractaire de notre politique, j'en étais persuadé, jusqu'au jour où un de mes collègues anglais m’a demandé : «Mais pourquoi avez-vous séparé l'infrastructure de l'exploitation ? Vous n'y étiez pas obligés par les textes européens. » J'ai pensé alors que, finalement, nous n'étions pas si réfractaires que cela. Sur certains points, nous sommes allés un peu plus loin que ce qui était strictement prévu par les textes. Évidemment, ça a été un petit peu compliqué, brouillé par les rapports bizarres entre la SNCF et RFF en matière d'entretien du réseau. Néanmoins, là nous sommes allés plus loin que la stricte obligation qui nous en était faite.

En matière de baisse du fret, je voudrais faire une petite mise en perspective. La part de fret en France était assez élevée, plus élevée que dans les pays voisins où elle a augmenté. Mais si elle a augmenté en Angleterre, il faut tenir compte du fait que les routes anglaises ne sont pas très propices à la circulation, elles sont assez congestionnées. De ce point de vue, si l'on adopte une perspective historique d'évolution sur de longues périodes, si on regarde les chiffres de part modale actuelle, la situation du fret n'est pas catastrophique. Elle l'est peut-être pour les comptes de la SNCF, mais elle ne l'est pas autant qu'elle pourrait le paraitre.

Ce qui me frappe aussi, c'est la diversité des choses que l'on met sous le même mot de «libéralisation » et « réformes ferroviaires ». Je vais peut-être un peu m'évader de l'Europe pour remarquer que dans tous les pays du monde, ou presque, il y a eu en quelques dizaines d'années des réformes ferroviaires, et que ces réformes ont pris des formes assez différentes. Finalement, ce n'est qu'en Europe que la réforme menée a conduit à une séparation de l'exploitation et de l'infrastructure. Aux États-Unis, il y a simplement eu une déréglementation, c'est-à-dire la disparition des réglementations de prix, de tarifs et de contingentement qui existaient. Au Japon, ce fut un découpage de l'entreprise nationale en sept parties géographiques qui ont été ensuite privatisées, mais la privatisation est venue ensuite, et n'a peut-être pas été l'élément le plus déterminant de la réforme. Pour élargir le champ, toutes les réformes menées par la Banque mondiale, notamment en Amérique du Sud, ont été fondées sur un découpage géographique des réseaux nationaux, puis sur une mise en concession sur de longues périodes des sections ainsi délimitées. Au Royaume-Uni, la réforme a été parallèle et allant au-delà, mais aussi quelquefois, finalement, contre la doctrine européenne, parce qu'au Royaume-Uni il 
n'y a pas de concurrence sur les rails : la réforme a allié un découpage complet que tout le monde connait avec la séparation de l'infrastructure et de l'exploitation et la décomposition des exploitants en un grand nombre d'opérateurs privatisés. Ce qui fait que finalement, la libéralisation, la réforme est passée par différentes composantes : la déréglementation, la privatisation, l'éloignement de l'État et puis des ersatz de concurrence, concurrence par comparaison au Japon, concurrence pour le marché en Amérique du Sud, et, en Europe, on ne peut pas qu'il y ait une concurrence parfaite. Ce sont des concurrences à la frange.

Par ailleurs, si on analyse les évolutions historiques en France, on ne peut pas dire que la libéralisation ait commencé en 1991. La libéralisation des transports et des chemins de fer a commencé pratiquement après la guerre lorsqu'il y avait des contingentements et une réglementation des tarifs routiers et également ferroviaires. Toutes ces contraintes se sont progressivement desserrées. Tout ce que l'on dit en termes de recherche d'une gestion commerciale, on le retrouve dans le rapport Nora, qui date de la fin des années 1960. Ce qui fait qu'il y a certainement une rupture dans les années 1990, mais je pense que, sur une très longue période, il y a aussi une très forte continuité dans le mouvement.

Pour répondre à la question d'Hubert du Mesnil concernant les TGV, je serais tenté de penser qu'il est heureux qu'on n'ait pas séparé plus tôt l'infrastructure de l'exploitation, parce que je crois que c'est l'intégration, précisément, de l'infrastructure et de l'exploitation, et les liens très forts entre l'opérateur et les constructeurs qui ont permis de mettre au point un système qui nécessitait une intégration technique forte. Je me dis que si cette séparation avait été faite beaucoup plus tôt, le TGV aurait peut-être eu plus de mal à voir le jour.

\section{Patrice Leroy}

Merci, Émile Quinet. Voulez-vous, Michèle, Merger donner à nouveau une perspective historique?

\section{Michèle Merger}

Je suis tout à fait d'accord pour souligner que la «thérapie de choc » et ces notions de libre concurrence, d'ouverture, etc., et les vertus de la concurrence ne sont pas liées à Bruxelles et qu'elles ne sont pas nées ex nibilo dans la tête de fonctionnaires européens. Ce sont des concepts et des approches de l'exercice de l'activité ferroviaire qui existaient dès le XIX ${ }^{\mathrm{e}}$ siècle, dès l'apparition des chemins de fer. Dans une première phase, les premières lignes venaient en complément des voies traditionnelles de transport du charbon de la mine vers le fleuve ou vers le canal, mais ensuite les chemins de fer se sont comportés 
comme toute entreprise qui a pour objectif de faire des bénéfices et du profit et d'avoir une attitude de conquête des marchés, de la clientèle du côté des voyageurs, et de conquête du trafic des marchandises.

Mais il faut bien reconnaître que la « thérapie de choc » est née d'un constat partagé par tous : à la fois par les entreprises ferroviaires traditionnelles, par de hauts fonctionnaires européens, et par ceux qui constataient l'évolution du trafic marchandises. C'est en voyant ce trafic décliner fortement, en part relative, comparé aux autres moyens de transport, la route en ce qui concerne le fret, dont on sait les vertus du point de vue de la flexibilité et le grand avantage du porte à porte, que cette thérapie de choc est née.

Il me semble aussi qu'il faudrait rappeler que les compagnies ferroviaires du XIX ${ }^{\mathrm{e}}$ siècle passaient des accords commerciaux et tarifaires, les fameux services communs transnationaux. On peut citer des exemples, ô combien célèbres, dans les domaines de voyageurs. Les travaux de Laurent Tissot ont mis en lumière cette stratégie commerciale des compagnies et les accords passés entre les compagnies anglaises et les compagnies françaises, compagnies de transit, pour acheminer les voyageurs anglais jusqu'aux sommets suisses. Or les directives sont en fait l'émanation d'un accord, des accords entre pays membres. Cette vision européenne, en matière de trafic de marchandises, s'exprime par les corridors de fret, qui reposent sur des accords qui correspondent à ceux que passaient les compagnies au XIX ${ }^{\mathrm{e}}$ siècle, c'est-à-dire un itinéraire élaboré par les opérateurs traditionnels, comme le corridor Belifret, et les freeways, que l'on peut rapprocher du concept d'autoroute, avec des opérateurs qui demandent des sillons et payent leur passage. La vision européenne de ces opérateurs est très évidente. Si, dans vingt ou quarante ans, un colloque est organisé dans le cadre de cette association, il faudra que les historiens de demain aillent consulter les archives de ces opérateurs auxquels on peut lancer un appel pour qu'ils les conservent de façon à ce qu'on puisse retracer leurs stratégies. Quand on fait de l'histoire au présent, on n'a pas toujours, et on ne peut pas avoir tous les éléments de réflexion pour écrire cette histoire qui se tisse sous nos yeux.

Je voudrais poser à nouveau quelques questions. M. Lebœuf évoquait, à propos du service public, les appels d'offres que les régions pourront lancer à partir de 2010. Cela sera-t-il plus facile dans le cadre des régions frontalières et de leurs liaisons transfrontalières ? Je pense notamment à l'Alsace, pionnière de la régionalisation dessinée par le sénateur Haenel. De fait, la géographie parle et efface les frontières entre réseaux ferroviaires. 


\section{Michel Lebœuf}

Dans ce domaine, les textes qui encadrent la procédure sont à la fois la loi SRU (solidarité et renouvellement urbain du 13 décembre 2000) et le règlement OSP. La loi SRU dit que la région organise le service national sur son périmètre propre, mais elle peut aussi organiser les relations TER avec la région adjacente et aussi avec sa voisine à l'étranger, au-delà de la frontière. Effectivement, on peut penser qu'il y aura des appels d'offres avec certains voisins qui, eux, sont beaucoup plus dans cet esprit. On peut imaginer, lorsque la liaison Barcelone-Perpignan sera réalisée complètement, que les régions Languedoc-Roussillon et Catalogne essaieront de mettre en place ce qu'on appelle le TERGV, le train régional à grande vitesse, c'est même tout à fait probable... tout au moins probable qu'il y ait un appel d'offres. Mais, parfois, il est assez difficile de faire des prévisions dans ce domaine. Aujourd'hui, le seul cas où l'idée d'un TER transfrontalier est apparue, c'est dans le Nord-Pasde-Calais, où la région veut faire une liaison TER entre Lille et Bruxelles. Il est probable que de telles coopérations se développeront.

\section{Yves Crozet}

Le transport régional est un aspect extrêmement important et, pour ma part, je crois beaucoup plus en l'impact de l'ouverture à la concurrence par les appels d'offres lancés par les régions sur une concurrence pour le marché, comme l'a dit Michel Lebœuf, qu'à l'idée de l'ouverture à la concurrence sur les trains internationaux. Si vous prenez Thalys, c'est quelque chose qui fonctionne bien et qui permet une interopérabilité entre plusieurs opérateurs. Il est beaucoup plus simple d'avoir un Thalys que trois opérateurs concurrents sur Paris-Bruxelles. Je ne crois pas du tout en l'efficacité de la concurrence dans ce domaine. En revanche, le fait que des régions puissent, pour tout ou partie de leur réseau régional, de leur exploitation régionale, faire appel à un autre opérateur que la SNCF est très intéressant, parce que les travaux qui ont été menés au Laboratoire d'économie des transports, je pense à Julien Leveque ou à Christian Desmaris ${ }^{5}$, montrent bien que la SNCF elle-même est un opérateur dont les performances sont très différentes dans les vingt et une régions françaises. On peut faire ce que nous, économistes, appelons de la concurrence par comparaison et démontrer quelles sont les régions où la $\mathrm{SNCF}$ est relativement performante et quelles sont celles où elle a profité des subventions régionales. Seule une université peut le dire ; j’en profite donc. On voit ainsi concrètement où sont les gains de productivité, où sont les surcoûts, où sont parfois les sureffectifs. C'était une première remarque.

5- Voir les pages personnelles des deux chercheurs sur le site du Laboratoire d'économie des transports : http://www.let.fr/fr/annuaire/auteurs/cdesmaris/index.php et http://www.let.fr/ $\mathrm{fr} /$ annuaire/auteurs/jleveque/index.php. 
Ma deuxième remarque porte sur le fret. La réponse à la question d'Hubert du Mesnil est très simple pour les économistes. Il suffit de lire les ouvrages d'Émile Quinet pour bien comprendre qu'il n'y a pas entre le fret et les voyageurs ferroviaires d'économie d'envergure. En d'autres termes, le fait de joindre les deux activités dans une même entreprise diviserait le coût moyen par deux. Il n'y a pas d'économie d'envergure comme il y en a dans l'aérien. Les grandes entreprises aériennes de transport de fret sont Fedex et UPS, et juste après elles vous trouvez des compagnies comme British Airways, Lufthansa et Air France, parce que, sous les voyageurs, il y a de la place et qu'on peut y mettre du fret. Sous les voyageurs des TGV, on ne peut pas mettre de fret. De plus, et cela a été très bien expliqué par Michel Lebœuf, la concurrence pour les voies est très importante. Par conséquent, la question est très délicate, parce que soit nous sommes devant une question d'organisation, et la solution est simple pour un universitaire : il faut séparer les deux activités, il faut sortir le fret de l'entité qui transporte les voyageurs pour qu'on ait deux entités différentes qui ensuite se battront auprès de RFF pour avoir des sillons, et pour que les péages soient calculés justement. Ou, plus grave, nous sommes devant un problème technique. Lorsqu'une voie donne systématiquement la priorité aux voyageurs, le fret est toujours perdant. C'est peut-être là le nœud du problème : si vous regardez les grands pays, mis à part sans doute la Chine et la Suisse aujourd'hui, partout où le fret est puissant, c'est que les voyageurs le sont très peu. En revanche la Suisse et la Chine sont deux pays où le fret et les voyageurs cohabitent très bien : en Suisse, on est à plus de $30 \%$ de parts de marché pour le fret ferroviaire - $35 \%$, en France, je vous rappelle qu'on était à $15 \%$. Et les Anglais étaient à $6 \%$ seulement après l'ouverture. S'il s'agit d'un problème technique, cela veut dire qu'il faut choisir clairement, et qu'il faut dire : «Désolés, on ne transporte que des voyageurs, parce qu'on a un beau système, et le fret, il sera limité à quelques lignes. » La question est vraiment très délicate, car c'est une question politique, et si j'osais, en tant que ministre, je ne dirais jamais qu'il n'y aura plus de camions sur les autoroutes dans les quinze ans, parce qu'on aura augmenté le fret ferroviaire. Mais je n'imagine pas qu'un ministre puisse dire une bêtise pareille.

\section{Patrice Leroy}

Une réaction, Michel, sur les régions, les négociations, la concurrence dans les régions? 


\section{Michel Lebœuf}

Je voudrais dire que, effectivement, le fait que le fret s'oriente vers une spécialisation des moyens est d'une certaine façon la reconnaissance de l'absence de ces économies d'échelle. Il n’empêche que le fret et les voyageurs circulent sur les mêmes voies. La question de l'allocation de la capacité entre fret et voyageurs est la question centrale. Et la question d'avoir des sillons fret de qualité est tout aussi centrale. Mais je sais que RFF s'en occupe et envisage même une tarification adaptée. En ce qui concerne la concurrence dans le domaine du transport de voyageurs, notre analyse est la suivante : effectivement, les contrats du service public que peuvent passer les régions avec des opérateurs sont les tremplins pour les opérateurs, parce qu'au fond ils ne prennent de risque que sur leur coût d'exploitation, le matériel roulant étant la propriété de la région. Du coup, il est beaucoup plus facile d'entrer dans le ferroviaire voyageurs par ce biais, que de franchir directement la grande marche pour accéder à la grande vitesse. Mais n'oublions pas que, dans le domaine de la grande vitesse, nous aurons certainement d'abord la concurrence des opérateurs historiques. Nous savons déjà par exemple que la DB souhaite exploiter Londres-Cologne. Ce ne sera pas facile, car, compte tenu des spécificités du tunnel, le matériel doit remplir un certain nombre de conditions. Il y a aussi plusieurs types d'alimentation électrique successifs, c'est un matériel qui n'est pas très simple, mais elle le souhaite. Air France s'est déjà exprimé en tant que futur opérateur ferroviaire, ça l'intéresse. Le cas le plus concret, c'est celui de NTV, Nuovo Trasporto Viaggiatori, qui est en train de passer commande d'un marché d'une quarantaine de rames à grande vitesse, dans l'intention de concurrencer TrenItalia en Italie, d'où nous pensons que ça risque de déborder en France, mais l'avenir nous le dira ${ }^{6}$.

\section{Émile Quinet}

Je voulais intervenir sur les remarques d'Yves Crozet et de Michel Lebœuf concernant la concurrence «voyageurs ». Je crois qu'en ce qui concerne les voyageurs, comme le disait Yves Crozet, on voit ce qui peut se passer avec les TER. Mais on est davantage dans le flou en ce qui concerne la grande vitesse et les précisions que tu as données vont dans ce sens. Je posais la question de la concurrence dans l'autre sens : qu'est-ce que la SNCF peut aller faire à l'étranger? Parce qu'il peut y avoir des réciprocités.

6- Précision apportée par Michèle Merger : derrière ce sigle, il y a Luca Cordero di Montezemolo, très connu en Italie pour être à la tête de Ferrari, et Diego Della Valle, un grand industriel, connu pour la marque de chaussures Tod's; ils veulent tous deux intervenir et faire en sorte que des trains à grande vitesse circulent sur les lignes à grande vitesse italiennes, lorsqu'elles seront construites, et qu'ils transportent des voyageurs sur le réseau classique. 


\section{Patrice Leroy}

J'aimerais qu'Hubert du Mesnil s'exprime, en particulier sur la régulation du système.

\section{Hubert du Mesnil}

Pour poursuivre nos échanges, je voudrais revenir sur un autre point et puis avoir aussi quelques échanges entre nous -, celui de la séparation de l'infrastructure dans le mode français pour donner un témoignage, car je pense quand même qu'il faut dire la vérité. Quand on a séparé l'infrastructure de l'exploitation en 1997, ce n'est pas parce que les décideurs politiques de l'époque croyaient en la vertu de la séparation de l'infrastructure ; si on l'a séparée c'est purement et strictement pour enlever la dette. Puisque, à peine avait-on enlevé l'infrastructure à la SNCF, on a dit : «Ne vous en faites pas, ça ne change rien, puisqu'on la redonne. » Le problème, d'ailleurs, de RFF, c'est bien un problème de cette nature, c'est-à-dire que le problème génétique du corps social du RFF, si je peux dire, biologique, c'est que c'est un être qui n'a pas été désiré.

Ce qui se passe c'est que, pour diverses raisons, il a fallu à cette période traiter prioritairement le problème du moment, le problème de la dette : on a inventé quelque chose qui permettait à la fois d'enlever la dette et en même temps de dire qu'on enlevait les actifs. Après, finalement, une fois qu'on l'a fait, un rapport à la Cour des Comptes dit - on sait pas si c'est à regret ou avec espoir - que RFF semble prendre goût à exercer vraiment les compétences pour lesquelles il a été créé. Il est vrai que l'enfant en grandissant s'est dit : «Après tout, pourquoi on ne ferait pas aussi un peu d'infra? » Je ne reviens pas plus longtemps sur ce thème. Mais il y a, à mon avis, un certain intérêt historique à procéder à une relecture de ce cheminement qui, vu de Londres ou de Bruxelles, n'est peut-être pas très facile à percevoir. Je veux dire qu'il y a un côté empirique dans notre démarche. Et je ne suis pas sûr que l'histoire se fasse exactement comme on l'aura imaginée ou voulue.

Je reviens sur la question fret/voyageurs puis sur la régulation du système. Pour moi, la question que je vous ai posée tout à l'heure, qui a été reprise par plusieurs intervenants, ne doit pas être perdue de vue parce qu'elle occupe beaucoup le fond de ma pensée. Il faudra rendre justice de cela et je retiens volontiers de ce qui a été dit que si la séparation de l'infrastructure avait été faite avant que la SNCF n'invente le TGV, je ne suis pas sûr qu'il y aurait le TGV. Nous rendons à la SNCF le fait que, sans doute parce qu'elle a été configurée comme ça, elle a su inventer le produit qui, probablement, n'aurait pu être inventé de la même manière si on n'avait pas eu cette configuration précise. 
Mais, ne peut-on dire aussi que c'est parce qu'elle a été configurée comme ça qu' a contrario elle n'a pas pu comprendre que le transport de fret et le transport de voyageurs sont deux métiers différents? Je veux aller un peu plus loin que ce qui a été dit: ce n'est pas uniquement une question économique, de synergie ou de l'économie d'échelle, c'est presque une question de stratégie d'entreprise, c'est que ce sont deux métiers différents. Et qu'une même entreprise se trompe et échoue quand elle ne sait pas quel métier elle exerce. Elle pouvait soit choisir d'en exercer un et de renoncer à l'autre, soit comprendre que ces deux métiers étant différents, il ne fallait pas les exercer de la même manière. Ce n'est pas parce que dans les deux cas on roule sur les mêmes voies ferrées qu'il s'agit du même métier. Comme j'ai eu l'occasion d'observer la route de très près, je me suis demandé s'il existe des entreprises, des autocaristes qui font le transport de marchandises ? Il est vrai que dans le mode aérien, Air France met les deux dans le même engin. Ils doivent donc utiliser cette économie-là pour apprendre à le faire. Mais il n'y a pas d'autocars dans lesquels on mette des marchandises, et il n'y a pas de camions dans lesquels on mette des voyageurs. Quand vous fréquentez le monde de la route, en l'occurrence notre mode concurrent, vous voyez que ce sont deux mondes complètement différents : leur structure syndicale, leur corps social, le personnel correspondant ne veulent pas qu'on les mélange parce qu'ils considèrent que ça n'a rien à voir. La même entreprise qui, parce qu'elle était intégrée, a su inventer le produit génial, n'a pas su voir, je pense, qu'elle se trompait de route en ce qui concernait le fret, c'est le cas de le dire. Je tenais à faire cette observation.

À propos de la question des sillons, de la gestion de la capacité d'abord, nous devons tous prendre acte du fait que de moins en moins de trains circulent en France. Notre métier étant de vendre du sillon, c'est-à-dire d'utiliser une capacité, nous sommes en régression permanente. Notre activité d'entreprise baisse sans arrêt, même lorsque la SNCF dit que depuis le début de l'année 2007, je mets entre parenthèses la période actuelle, il y a eu un redressement de l'activité fret. Certes, ce redressement de l'activité fret existe pour la SNCF, mais il n'existe pas pour RFF. Le nombre de trains qui circulent n'augmente pas. Notre activité étant de compter des trains, bien sûr sans perdre de vue ce qu'ils transportent, nous comptons, nous vendons la capacité, nous voyons que le nombre de trains continue à diminuer : l'activité ferroviaire en France n'a pas cessé de diminuer depuis une quinzaine d'années. Nous sommes donc toujours dans la régression. C'est ça qu'il faut voir en face. Dans nos prévisions de moyen terme, nous nous demandons si, un jour ou l'autre, en France le nombre total de trains augmentera. Évidemment, c'est le fret qui est la clé de la réponse, puisqu'on ne doute pas que le nombre de TGV puisse augmenter au fur et à mesure des investissements. Mais quand on ne crée pas 
de lignes nouvelles de TGV, le nombre de TGV n'augmente pas. Le nombre de voyageurs augmente, parce qu'on double le TGV en longueur et en hauteur, mais le nombre de trains n'augmente pas. Donc, il reste de la capacité, puisque le nombre de trains est diminué. Il ne faut pas se faire de souci. Si un jour on arrive à faire rouler plus de trains, ne vous en faites pas, on a tout ce qu'il faut : le réseau est surabondant. Je sais bien qu'il y a quelques nœuds dont il faut s'occuper, avec le contournement de Lyon ou autre... Mais au total, ça passe.

Ensuite, il faut que le système ou l'autorité qui distribue les sillons, quand il reçoit deux demandes, d'une part ait le pouvoir de les rendre compatibles, d'autre part ne fasse pas un choix purement économique. Si on regarde le comportement d'entreprise à Réseau ferré de France, il vaudrait mieux que lorsqu'on vend des sillons à une entreprise qui transporte du fret ça ne nous fasse pas perdre de l'argent chaque fois que le train roule, auquel cas moins il roule, mieux on se porte. Il y a bien un système à trouver, on a une idée, on l'a proposée, allons-y, continuons! Sous cette seule réserve-là, qui peut être levée très facilement, il suffit de le vouloir, et si l'État le veut, on l'applique très vite, on ne demande pas d'argent supplémentaire, on propose seulement d'affecter la ressource publique pour compenser le décalage entre le coût réel du passage du train et le coût supportable en fonction du marché. Bruxelles ne nous empêche pas de le faire. Et même si elle nous en empêchait, on le ferait, on trouverait le moyen de le faire. Et je pense qu'on sera dans l'esprit de développement durable, ça ira très bien. Sous cette réserve-là, ça va bien.

Simplement nous, en tant que distributeur de capacités, nous aurions besoin de partager avec les autorités publiques, parce que nous considérons que nous ne sommes pas les seuls à donner la réponse, la manière de répartir cette capacité entre les uns et les autres. De ce point de vue, le problème nous dépasse nous, entreprise, car c'est un véritable problème national : est-ce que, par exemple, nous pourrions partager avec les responsables politiques, je pense notamment aux présidents de régions, le fait que c'est aussi leur problème que des trains de fret roulent ? C'est aussi leur problème, qu'on arrive, pas tout de suite, mais assez vite, à choisir, à réguler, en disant sur cette voie je fais passer $\mathrm{X}$ trains de voyageurs et $\mathrm{Y}$ trains de fret dans telles conditions. Cela veut dire qu'au dernier train de voyageurs qui voudrait être créé, on dira «non », parce que, entre telle heure et telle heure, on a réservé les capacités pour le fret. On n'en est pas encore là. C'est vrai, je retiens tout à fait ce qui a été dit, nous regrettons que dans les réflexions actuelles sur le régulateur, on n'ait pas encore créé en France une capacité d'arbitrer ou de décider à un moment donné que, pour faire passer l'ensemble des trains, il faut répartir cette capacité, si on approche de la saturation ; ce n'est pas nous, Réseau ferré de France, qui devons faire cet arbitrage en fonction d'un intérêt politique ou de notre préférence. 
Ce n'est pas non plus le rapport de forces qui existe entre les entreprises ferroviaires, parce que l'une crie plus haut que l'autre. C'est un élément de responsabilité publique que nous voudrions voir prendre en mains par une autorité publique.

\section{Michèle Merger}

Une question à M. du Mesnil, à propos de la tarification de l'infrastructure. La taxation doit suivre le coût marginal, mais il existe quand même des coûts externes : le bruit, etc. C'est un vrai débat, qui dépend en fait des États membres. Or l'absence d'une autorité de régulation en France ne pose-t-elle pas un problème supplémentaire à RFF de ce point de vue ?

\section{Hubert du Mesnil}

Je pense qu'en la matière, presque tout est devant nous, parce que le modèle tarifaire à appliquer à l'infrastructure ferroviaire devrait être bâti à l'échelle de l'Europe, et qu'il ne l'est pas du tout. Je pense qu'il devrait être ensuite harmonisé au niveau intermodal, pour que cette prise en compte de coûts externes, par exemple, puisse être faite, pour nous, comme pour d'autres modes. Ça pourrait par exemple se traduire par le fait que lorsqu'il y a des sections qui engendrent du bruit, on fasse payer plus cher ; lorsqu'il y a des zones très saturées, dans les réseaux denses, on fasse payer plus cher, etc. En contrepartie, on internalise d'autres avantages sur le plan économique. Actuellement, dans nos évaluations économiques, on met très peu de valeurs écologiques. Ça a été déclaré abondamment à l'occasion du Grenelle de l'environnement, mais c'est vrai. Simplement, on ne peut progresser qu'en tenant compte d'une vision européenne, parce que nos concurrents vont venir sur le réseau et on n'imagine pas qu'on puisse leur imposer une tarification qui ne soit pas lisible pour eux qui viennent de l'étranger, et qu'ils puissent comparer ou comprendre chez nous par rapport à ce que font les voisins. Il s'agit bien d'un problème d'harmonisation des règles de tarification européenne pour le mode ferroviaire. De même qu'il y a simultanément un problème d'harmonisation de la tarification intermodale entre nous et la route. Tout est devant nous dans cette affaire, et on ne fait que répéter inlassablement qu'il est temps, plus que temps, que la France s'en saisisse.

Je suis très prétentieux, excusez-moi, mais je propose qu'à la prochaine présidence française de l'Union européenne, la France, qui a beaucoup d'ambition en ce moment vis-à-vis de l'Europe, on l'a bien senti, mette sur la table cette question de la tarification européenne, intermodale. Vous savez qu'il va falloir qu'on relance le dossier de l'euro-vignette, et les autres États sont assez satisfaits que cette idée ait progressé ces dernières semaines avec le Grenelle de 
l'environnement. Il me semble qu'on serait plus forts pour faire passer cette euro-vignette routière européenne, si, dans le même temps, on mettait en chantier la tarification ferroviaire. Cela permettrait de faire avancer et l'un et l'autre sous une même autorité, et, puisqu'on vient de marquer un point avec cette euro-vignette ou le principe même de cette redevance routière, je trouve que ça aurait du sens, que ce serait équilibré de dire que dans le même temps il faut avancer sur la voie de la tarification ferroviaire.

Il reste les débats que nous avons sur le niveau de la tarification de l'infrastructure. Je peux, si vous le voulez, pour alimenter notre table ronde, essayer de vous dire ce que nous en pensons. Je voudrais profiter de mon expérience, ayant traversé successivement les différents modes, pour dire qu'il est parfaitement normal que le sujet de la tarification soit un sujet de confrontation entre le gestionnaire d'infrastructure et le transporteur. Quand on me dit : «Il faut arrêter de vous battre avec la SNCF », ça n'a pas de sens. J'entendais la même chose quand j'étais à Aéroports de Paris : "Arrêtez de vous battre avec Air France! » C'est un sujet de contradiction. Il faut le prendre comme tel. Dans un cas, une situation de monopole, nous, gestionnaires d'infrastructure, qui, évidemment, n'avons en tête qu'une chose, faire payer le plus cher possible. De l'autre côté, une entreprise qui est en concurrence avec d'autres concurrents du même mode ou avec des modes concurrents, qui n'a qu'une peur, celle de se faire étriller par le gestionnaire qui, lui, est en position de monopole. Une telle situation est forcément conflictuelle. Il est absurde de dire qu'il faut arrêter de se battre. En revanche, il faut essayer de se battre intelligemment. Puisqu'il y a une confrontation, il faut qu'il y ait un dialogue, il faut qu'il y ait un troisième acteur, le régulateur ou l'État, qui ait le pouvoir de nous faire jouer intelligemment ce jeu, de nous pousser dans nos retranchements. Il faut qu'il y ait des économistes, des observateurs et des experts qui nous disent : «À quoi ça rime, votre tarif ? Vous voulez payer quoi ? Qu'estce que le coût marginal ? » Il ne doit pas s'agir d'un face à face où l'on se regarde en se chipotant, mais d'une confrontation à deux, à laquelle participent d'autres acteurs qui ont leur mot à dire pour éclairer le débat, pour lui donner du sens, pour comprendre la réalité économique de ce dont nous parlons. Il faut également un acteur décisionnaire qui lui, à un moment, dise : «Stop! Maintenant, c'est comme ça. » Ce n'est pas encore le cas en France. Il y a encore du chemin à faire pour que cette confrontation s'organise intelligemment et que, par ailleurs, on puisse avoir une politique tarifaire compréhensible et qui ait du sens, étant arbitrée finalement par l'État, parce que c'est bien son rôle. Cette confrontation étant normale, nous pensons qu'il en va de l'intérêt du système ferroviaire et de ses utilisateurs, donc des entreprises. Contrairement à ce qu'elles semblent dire, il est dans leur intérêt que ce 
système engendre lui-même sa propre richesse. Plus l'infrastructure pourra être payée par ses utilisateurs, plus cela voudra dire que le système ferroviaire créera de la valeur économique, et mieux il se portera. Je me retranche derrière les experts et les économistes, mais c'est notre conviction. Ce n'est pas faire de la peine à la SNCF que d'aspirer à ce qu'elle paye de plus en plus cher l'infrastructure, parce que cela signifierait qu'elle vaut quelque chose. D'une certaine manière, lorsque le fret ferroviaire en France pourra payer l'infrastructure, ça voudra dire qu'il est sauvé.

\section{Patrice Leroy}

Merci, tout cela soulève beaucoup plus de questions que nous n'aurons le temps d'en traiter. Yves Crozet, que pouvez-vous dire sur toutes ces questions soulevées à la fois : le modèle ferroviaire européen, le coût externe, la place du régulateur, le risque d'une situation oligopolistique comme dans l'énergie ou la communication?

\section{Yves Crozet}

Je crois qu'on peut en effet résumer ce qu'a dit Hubert du Mesnil de façon un peu caricaturale, en disant qu'une façon intelligente de se battre consiste à faire payer l'utilisateur. Je souscris tout à fait à cette vision. Je pousse loin la caricature, mais l'idée selon laquelle l'ouverture à la concurrence sur les grandes lignes ferroviaires TGV est indispensable pour faire baisser le prix pour l'utilisateur ne doit pas être répandue. Parce que, actuellement, dans le système ferroviaire, ce que paye la SNCF à RFF sur certaines lignes, par exemple LyonParis, est très supérieur au coût marginal. En revanche, ce qui est payé sur Paris-Strasbourg est inférieur non pas au coût marginal, mais au coût total, puisqu'il a fallu subventionner l'exploitation. Si on veut développer le système ferroviaire à l'échelle française, le président de la République a parlé de $2000 \mathrm{~km}$ de lignes nouvelles, il est évident que cela doit se faire avec une participation sensible de l'usager. Rappelez-vous que, quand la première ligne à grande vitesse a été ouverte en septembre 1981, le prix du billet de seconde classe Lyon-Paris était le même que le prix du billet sur la ligne classique. Quand on ouvre Paris-Strasbourg en juin 2007, le prix du billet Paris-Strasbourg de seconde classe en TGV est d'emblée 20 euros plus cher que le billet de train classique. Les usagers et leurs élus ont bien sûr protesté. Mais il ne faut pas être sensible à cette protestation, puisque c'est le but du jeu. Soyons clairs : les calculs économiques peuvent nous le montrer sans que l'opérateur SNCF se comporte comme un rentier qui augmentera les prix à un niveau si élevé que le trafic s'effondrera, puisque ce n'est pas ce qui s'est passé. Tout le monde 
s'insurge contre les prix trop élevés, les trains sont pourtant pleins quand ils circulent. La demande est extrêmement forte, et cette demande forte, il faut la faire payer. Si on veut un jour avoir un TGV PACA, même sur une partie de la ligne, il devra être subventionné pour son infrastructure à hauteur de 60-70$80 \%$, mais ça ne doit surtout pas être une raison de la réaliser à bas prix. Il faut absolument que ces TGV soient à des prix relativement élevés. C'est aussi parce que le TGV verra au moins une partie de son coût payé par les voyageurs qu'on pourra dans le même temps justifier les taxes sur le kérosène pour les avions, qu'on pourra justifier les taxes sur les poids lourds, etc. Il ne faut surtout pas laisser penser, comme on l'a fait pour l'énergie, qu'« on va libéraliser l'énergie et vous payerez l'énergie moins cher »... Quand on sait ce qu'il va falloir investir dans les années à venir dans l'énergie, il est stupide de parler de baisse des tarifs. Pour le téléphone, c'est autre chose, car pour ces réseaux le problème est tout à fait différent, mais pour l'énergie, pour le transport, ce serait une stupidité. Il va falloir que les usagers payent plus, et pas seulement pour des questions de $\mathrm{CO}_{2}$.

\section{Michel Lebœuf}

Hubert du Mesnil a dit que la confrontation était inévitable, mais constructive. Je crois qu'on ne va pas s'en priver maintenant. D'abord, je voudrais rectifier une chose. Le TGV ne paye pas que son coût marginal, il paye le coût complet, c'est-à-dire les charges financières, la construction... Il est vrai que le nombre de trains n'augmente pas, vu de RFF, mais les revenus de RFF augmentent. Et l'on peut s'en féliciter, parce que d'un côté les revenus de RFF augmentent, en tout cas pour le TGV, c'est tout à fait vrai, et de l'autre c'est la preuve que le chemin de fer est capable de massifier les flux, puisque le trafic augmente, en tout cas récemment. Donc, si le nombre de trains n'augmente pas, c'est que le poids par train augmente. C'est une très bonne chose.

Maintenant quand on parle du niveau des péages, je suis très inquiet à propos du péage des TGV, parce qu'on a fait quelques calculs sur la base des hypothèses formulées dans le dernier rapport du Conseil général des Ponts et Chaussées et de l'Inspection générale des Finances. Si l'on prend, par exemple, une relation comme Paris-Nice en rame duplex à une heure normale, pas en heure de pointe, elle roule en unité simple, remplie à $65 \%$, le péage par voyageur sera, en 2015, de 44 euros (88 euros aller-retour). Maintenant, quand on s'interroge sur le tarif Easy Jet, par exemple, par Internet, pour les six semaines qui viennent. C'est-à-dire que je pose la question pour aujourd'hui, pour demain, pour après-demain pour dans un mois et demi pour connaitre la moyenne du tarif Easy Jet. J'obtiens la somme de 119 euros l'aller-retour. Le péage sera à 88 euros, alors que le prix du billet sur Easy Jet sera à 119 euros 
- et la SNCF n'a pas que le péage comme coût : elle doit acheter des rames, il lui faut des conducteurs, entretenir ses rames... Donc, le niveau de péage, dans de nombreux cas, va atteindre des niveaux relativement inacceptables. Globalement, dans le rapport de l'IGF et du CGPC qui adopte une approche que je trouve extrêmement budgétaire, ça fonctionne. Mais quand on regarde marché par marché, ça ne fonctionne pas. J'attire l'attention sur le fait que nous avions réussi à contingenter le trafic des compagnies aériennes low cost, voire à éliminer ces compagnies, mais que nous allons revenir en arrière. Je signale un fait assez extraordinaire : la France est en Europe un des pays où les distances sont les plus longues avec l'Espagne et l'Italie. Mais nous sommes le pays où il y a le moins de compagnies aériennes low cost. Pourquoi ? Parce que c'est nous qui sommes la compagnie low cost. Si les péages augmentent trop, nous cesserons de l'être. Il n'y aura plus de raison pour que les compagnies aériennes low cost ne s'installent pas. Aujourd'hui, par exemple, le trafic de nuit qui part de Barcelone par la compagnie Lypsos devient très déficitaire, notamment à cause des péages. Nous sommes très impatients de voir la nouvelle ligne entre Barcelone et Perpignan mise en service parce que nous avons du mal à survivre et la réalité est qu'il faudra arrêter cette exploitation. Ce qui fait que le RFF aura encore moins de trains.

Il y a un point sur lequel je rejoins tout à fait ce qui a été dit sur les péages au niveau européen. Je pense que, dans la politique européenne, plusieurs volets ont été souhaités par l'Europe : le volet du désendettement, le volet de la libéralisation, et un troisième volet très important, le volet de l'interopérabilité. Je pense que, dans ce domaine, des efforts très importants ont été faits par la Commission européenne, des efforts importants seront faits par les gestionnaires de l'infrastructure et les entreprises ferroviaires. Mais quelque chose me choque profondément, c'est qu'en matière d'interopérabilité, en même temps qu'on fait des efforts coûteux au plan technique, on est en train de créer au niveau de la tarification quelque chose de totalement aberrant. On s'est amusé dans une étude faite assez récemment à voir comment les sillons sont tarifés en Europe par les différents gestionnaires de l'infrastructure. En gros, ils utilisent une quarantaine de paramètres différents. Chaque gestionnaire de l'infrastructure en utilise six ou sept, mais ce ne sont pas les mêmes. Lorsque vous passez d'un pays à l'autre, vous êtes incapable de prévoir quel sera le péage de bout en bout. En tout cas, vous êtes incapable d'en avoir un ordre de grandeur par un calcul mental, ce que j'appelle une tarification inintelligible. Sans compter qu'il y a dans l'infrastructure une très grande instabilité des péages. Si je prends le cas de RFF, nous avons vu d'une année sur l'autre que le tarif N2, par exemple, a augmenté de 38 à $40 \%$. Et ça va continuer sans compter qu'il peut y avoir des changements dans la structure. Si c'est la même chose 
dans les autres pays, l'opérateur qui a envie d'investir dans du matériel roulant pour pouvoir exploiter les relations internationales commencera par se dire : "Mais alors, quels vont être les péages dans dix ans ?", parce qu'il ne peut pas rentabiliser le matériel sur un an. Je souscris donc tout à fait à l'idée qu'il y a vraiment quelque chose à faire au niveau européen à la fois en termes de philosophie de coût (coût marginal ? coût moyen ? coût complet ?), en matière d'internalisation des effets externes et puis, également, un minimum de convergence sur les paramètres, ce qui permet de calculer le coût d'un sillon. Sinon, le système est complètement incohérent.

Du débat avec la salle, nous avons retenu le témoignage de M. Michel Walrave, directeur général adjoint de la SNCF puis directeur général de l'Union internationale des chemins de fer.

\section{Michel Walrave}

Ce n'est pas tant une question qu'une remarque et une contribution à verser au débat qui a été lancé par Hubert du Mesnil. Pourquoi la SNCF a-t-elle réussi le TGV et pourquoi a-t-elle échoué dans les marchandises ? Michel Lebœuf a donné une explication : la mythologie de la vitesse. Pour ma part, je n'y crois pas beaucoup. Je pense qu'il y a deux éléments fondamentaux, d'une part, le marché, d'autre part, la technologie.

Le marché. Hubert du Mesnil avait tout à fait raison de dire que les marchandises et les voyageurs, ce sont deux métiers totalement différents. Le marché des marchandises s'est extrêmement éclaté : chaque chargeur, chaque marchandise a ses problèmes spécifiques d'exigence de qualité de service, de matériel, etc. En revanche, les voyageurs donnent une stabilité à long terme au marché, la population des plus grandes agglomérations croît... Je prends un autre exemple à propos des marchandises : il ne faut pas oublier que quand Louis Armand a fait l'électrification en 25000 volts sur la ligne ValenciennesThionville, il a d'abord pensé aux marchandises, ce qui prouve qu'à l'époque l'idée des marchandises prévalait, pour acheminer le trafic du minerai de fer de la Lorraine vers la sidérurgie du Valenciennois. J'ai vécu la période, vingt ans plus tard, où le trafic de minerai venait du port de Dunkerque pour alimenter la sidérurgie de Lorraine. Cela montre bien l'instabilité à long terme du trafic « marchandises ». C'est une incidente.

En matière de marchandises, la SNCF, mais aussi la Deutsche Bahn, a beaucoup réfléchi à des systèmes techniques, automatisations de transfert de containers, des wagons automoteurs, etc. Et aucune solution technologique 
n'a été trouvée, parce que, je crois, il n'y en a pas, ou alors, elles sont très coûteuses et incompatibles avec les conditions de concurrence sur le marché. Je crois que l'explication est là et qu'elle est très simple.

Je rappelle un souvenir très précis pour dire que la SNCF n'a pas toujours misé sur les voyageurs. Quand je suis entré dans cette maison, en 1964, pratiquement tous les patrons de l'époque, à l'exception d'un seul, Roger Hutter, m'ont dit: «L'avenir du trafic ferroviaire, l'avenir du chemin de fer, ce sont les marchandises. Les voyageurs, il faut les oublier. Regardez ce qui se passe aux États-Unis : c'est ce qui nous attend dans 10 ans, 15 ans, ou 20 ans. » Il y avait quand même une idée très ancrée selon laquelle l'activité des marchandises était l'activité de base du chemin de fer à long terme. Évidemment, l'évolution des structures des marchandises à transporter, le développement de la concurrence routière dont les progrès ont été certainement sous-estimés ont fait changer les choses. Il y a aussi, je crois, un phénomène plus général, c'est que l'Europe est un continent qui se prête beaucoup moins au trafic marchandises que les États-Unis, sans aller prendre des exemples comme la Chine ou l'Inde. L'Europe est un continent dont les côtes sont très découpées, or avec le développement de la mondialisation, une grande partie des transports sont des transports complémentaires du transport maritime et, de tous les points du territoire de l'Europe, presque aucun n'est éloigné de plus de $500 \mathrm{~km}$ d'un port. À mon avis, c'est ce facteur qui joue contre le développement du trafic ferroviaire, sauf révolution du côté des coûts externes, etc. 


\section{ANNEXE \\ Chronologie de la libéralisation ferroviaire 1991-2004 par Michèle Merger}

Directive 91-440 du 29 juillet 1991 relative au développement des chemins de fer communautaires. Quatre changements fondamentaux sont envisagés :

- indépendance de gestion,

- séparation de la gestion des infrastructures et de l'activité de transport,

- assainissement financier,

- accès à l'infrastructure permis à des tiers.

Cette directive est complétée par les deux directives du 19 juin 1995 :

- directive 95/18 ayant pour objet la licence des entreprises ferroviaires ;

- directive 95/19 concernant la répartition des capacités d'infrastructure ferroviaire et la perception des redevances d'utilisation de l'infrastructure. Le droit d'accès est limité à tout regroupement international.

\section{Directive 96-48 du 23 juillet 1996}

Elle prévoit l'interopérabilité du réseau européen à grande vitesse.

\section{Premier paquet ferroviaire du 26 février 2001 (Paquet infrastructure ferroviaire), entré en vigueur le 15 mars 2001}

C'est un ensemble de trois directives qui met en place des mécanismes visant à favoriser une meilleure intégration du marché ferroviaire.

\section{Directive 2001-12}

Elle modifie la directive 91-440 et pose le principe du droit d'accès équitable des entreprises ferroviaires au réseau transeuropéen de fret ferroviaire (RTEFF) qui regroupe les principales voies des États de l'Union $(50000 \mathrm{~km}$ soit un tiers de la longueur totale des réseaux européens, sur lesquels circulent $70 \%$ du trafic marchandises).

Ce principe devra être étendu à toutes les lignes à partir de 2008.

Elle améliore la transparence et les garanties de la concurrence. Elle impose de séparer les comptes des trafics marchandises des comptes des dessertes voyageurs ; les subventions publiques ne doivent pas être croisées : l'aide publique au service voyageurs déficitaire ne doit pas être détournée et financer une baisse des prix du fret visant à élargir des parts de marché. 


\section{Directive 2001-13}

Elle modifie la directive 1995-18; elle élargit la notion d'entreprise ferroviaire et prévoit que les licences (sillons de circulation) et les taxes d'accès à l'infrastructure (péages) doivent être décidées par un organisme indépendant des exploitants ferroviaires.

\section{Directive 2001-14}

Elle modifie la directive 95-19 et prévoit que les chargeurs peuvent demander des capacités d'infrastructure sur leur propre territoire. Cette directive est applicable depuis le 15 mars 2003.

\section{Directive 2001-16 du 19 mars 2001}

Elle prévoit l'interopérabilité des trains ordinaires.

Deuxième paquet ferroviaire proposé le 23 janvier 2002 (Vers un espace ferroviaire intégré) et adopté le 16 mars 2004

Il vise à accélérer la création d'un espace ferroviaire intégré et propose des actions qui avaient déjà été envisagées dans le Livre blanc La Politique européenne des transports à l'horizon 2010 : l'heure des choix, publié en septembre 2001.

- Développer une approche commune de la sécurité ferroviaire.

- Compléter les principes fondamentaux de l'interopérabilité.

- Création d'une Agence ferroviaire européenne pour mettre au point des solutions communes en matière de sécurité et d'interopérabilité.

- Ouverture de l'ensemble du transport international du fret y compris le cabotage au $1^{\text {er }}$ janvier 2007 (et non au $1^{\text {er janvier }} 2008$ comme le prévoyait la directive 2001-12).

- Adhésion à l'Organisation pour les transports internationaux ferroviaires (OTIF).

\section{Présentation du troisième paquet ferroviaire le 3 mars 2004}

La Commission envisage :

- l'ouverture à la concurrence des trafics internationaux de voyageurs en 2010 ;

- la certification des conducteurs de locomotives et de trains (compétences spécifiques requises);

- la définition des droits des passagers (ponctualité, moyens de recours, remboursements) en transport ferroviaire international ;

- l'amélioration de la qualité des services de transport de marchandises par chemins de fer. 